\title{
Distribución local de la inversión de decisión regional: el caso del FNDR en Chile 1997-2012
}

\author{
José Miguel Muñoz \\ Universidad de Chile
}

\section{Resumen}

Esta investigación busca determinar los factores que inciden en que una comuna tenga una mayor o menor asignación de recursos vía proyecto FNDR, dentro de las regiones de Chile, para el período 19972012, a través de una metodología cuantitativa. Se concluye que si bien el FNDR es eficaz en propender a una equidad interregional, no lo es al momento de propender a una equidad intra-regional, lo que abre espacio para la innovación institucional.

Palabras clave: Inversión Regional, desarrollo local, proceso de toma de decisiones.

Local distribution of regional decision investment: the FNDR case in Chile 1997-2012

\begin{abstract}
This research seeks the factors that contribute a commune to have a greater or lesser resources allocation from the FNDR project within the regions of Chile, for the period 1997-2012, through a quantitative methodology. We conclude that although the FNDR is effective in tending to interregional equity, it is not effective in the search of intra-regional equity, opening space for institutional innovation.
\end{abstract}

Keywords: Regional investment, local development, decision-making process.

*Dirección de correspondencia [Correspondence address]: José Miguel Muñoz, Universidad de Chile

E-mail: asdf.jmimm@gmail.com 


\section{Introducción}

La literatura tiende a relacionar fuertemente desarrollo local y descentralización, debido a la necesidad de generar territorios competitivos, que aprovechen sus recursos locales endógenos, generando economías que aprovechen las potencialidades locales, que se complementen con políticas nacionales de desarrollo (Silva, 2003) y que puedan insertarse en "cadenas globales de valor" (World Trade Organization et al., 2011). Los factores clave, en un contexto de globalización, deben ser los distintos tipos de capital de carácter inmaterial, vinculados al conocimiento formal o tácito (Boiser, 1999)

El análisis empírico de la evolución económica regional entre territorios para seis países latinoamericanos en el período 1990-2003 cruzando crecimiento económico con riqueza (Silva, 2003), da como resultado la caracterización de cuatro tipo de territorios, evidenciando la vinculación entre disparidades regionales con la incapacidad de las economías regionales "periféricas" para utilizar sus recursos naturales y reconvertir sus economías desde sectores poco productivos y tradicionales a otros más dinámicos. El análisis en detalle para Chile muestra que las disparidades se dan entre regiones productoras de materias primas de mercado emergente (norte de Chile) y productoras de materias primas de mercados en decadencia (sur de Chile) (Silva et al., 2012).

La descentralización ha sido planteada en América Latina como un proceso de reforma del Estado que permitiría corregir estas disparidades al fomentar el desarrollo económico local con equidad entre distintos territorios sub-nacionales, aumentando la eficiencia en la asignación y producción de bienes y servicios y la participación social-ciudadana en la gestión del Estado. De este modo, democratización y eficiencia han sido las principales metas de la descentralización en América Latina (Finot, 2002).

En el contexto chileno, y de forma creciente, se ha puesto énfasis en la relación entre un excesivo centralismo y una dinámica de desarrollo económico territorialmente desequilibrado, altamente inequitativa y poco participativa (Palma, 2009). El funcionamiento administrativo altamente centralizado del Estado hace que existan múltiples obstáculos institucionales al desarrollo de instrumento pertinentes para una estrategia regional integrada de desarrollo. Los municipios, sobre todos en comunas de escasos recursos, tienen capacidades técnicas limitadas, siendo dependientes de las transferencias del nivel central y regional para la realización de proyectos de inversión orientados a sectores estratégicos en sus planes de desarrollo comunal, cuya aplicación es exigua. Mientras que los gobiernos regionales tie- nen amplias funciones, estas no se condicen con sus atribuciones y recursos (Insunza, 1997).

El Fondo Nacional de Desarrollo Regional (FNDR) es el principal instrumento a través del que se ha dotado de recursos a las regiones para el desarrollo de distintas iniciativas de inversión con relativa autonomía político-administrativa en la priorización y toma de decisión, representa la mayor parte de la Inversión de Decisión Regional, siendo llamado "el fondo de la descentralización" (Schiling, 1999). Sin embargo, existe poca claridad respecto a los factores que explican una mayor o menor inversión en determinadas comunas y regiones respecto a otras. Como resultado, los proyectos asociados al FNDR terminan sirviendo muchas veces de suplemento presupuestario para la inversión municipal o siendo asignados según un criterio de compensación territorial que no considera la eficiencia del gasto (Insunza, 1997) o por criterios eminentemente políticos (Acuña, 2009).

El estudio de la distribución del FNDR, y de los criterios con que este se asigna, es particularmente importante a la luz de los debates presentes sobre descentralización, tanto en el poder ejecutivo como legislativo, siendo aún incipiente el debate legislativo sobre las funciones, atribuciones e instrumentos que estos cuerpos colegiados deben tener para realizar un diseño, coordinación e implementación de política que facilite, y no obstruya, el desarrollo local.

La generación de evidencia empírica, respecto a los criterios con los que actualmente se asignan los proyectos de inversión en el FNDR en las comunas, nos permitiría comprender mejor nuestra institucionalidad y detectar posibles problemas y nudos críticos. Así, el objetivo de la investigación es determinar los principales factores económicos, sociales e institucionales que explican la forma en que se distribuye el Fondo Nacional de Desarrollo Regional en Chile entre comunas de una misma región, desde 1997 al 2012.

\subsection{Descentralización}

El concepto de descentralización se ha utilizado de diversas maneras y desde distintas dimensiones (Von Haldenwag, 1990). Prud'homme (1995) habiéndose distinguido entre tres procesos distintos: i) Descentralización Espacial, caracterizada por la difusión poblacional y de actividades económicas desde los centros demográficos hacia zonas geográficas de menor densidad; ii) La liberalización económica, entendida como el traslado de la producción de bienes y servicios desde el Estado al Mercado; y iii) La descentralización administrativa, entendida co- 
mo la transferencia de responsabilidades desde el gobierno central a las unidades de campo del mismo gobierno (Rondinelli, McCullog, \& Johnson Donald, 1989)

La descentralización administrativa remite específicamente a la estructura del Estado, refiriéndose a la distribución de diversas funciones del mismo entre las distintas agencias gubernamentales y al grado de autonomía que estas distintas agencias tienen para la toma de decisión respecto a sus funciones. La descentralización administrativa es un concepto que remite a un proceso político-económico altamente complejo dentro del Estado que condensa variables institucionales, partidarias, financieras, y administrativas. Sin embargo, sólo para fines analíticos, es posible distinguir dos dimensiones de la descentralización administrativa: la dimensión política, referida a la generación de autoridades y sus procesos de decisión; y la dimensión fiscal, referida a los mecanismos de financiamiento y gasto.

La Descentralización Fiscal se refiere a los desplazamientos de competencias fiscales (ingresos y gastos) desde el nivel central del Estado a niveles inferiores o subnacionales (Aghón, 1993, 2001). Respecto a los ingresos, se pueden identificar tres formas principales de financiamiento de las unidades subnacionales: 1) Impuestos cobrados por las unidades descentralizadas; 2) Transferencias Intergubernamentales, desde el gobierno central a las unidades descentralizadas; 3) Acceso al crédito por parte de las unidades descentralizadas (Monasterio y Suárez, 1998). En el caso de las transferencias, existe una amplia gama de posibilidades y variantes de transferencias, pudiendo ser condicionadas, cuando el gobierno central determina el fin para el que deben utilizarse dichos recursos; o de libre disposición, cuando no existe una contraprestación o condicionante asociada a la transferencia. Si la transferencia es condicionada, la condición impuesta puede ser de carácter general, cuando es acotada a un sector específico de política pública; o de carácter específica, cuando se refiere particularmente a la provisión de un bien en específico, o la realización de un proceso determinado. Mientras que las transferencias no condicionadas pueden ser realizadas en base a un grado de participación en los recursos generales del Estado, o en base a algún criterio de compensación a los gobiernos subnacionales (Monasterio y Suárez, 1998).

Respecto a los efectos de la Descentralización Fiscal, existen diversos puntos de vista que apuntan tanto a las ventajas como a las desventajas de la misma (Letelier, 2012). Una de las principales desventajas tratada por Prud'homme (1995), es el posible impacto negativo que puede tener la descentralización fiscal sobre la equidad, particularmente sobre el grado de redistribución o progresividad del gasto total del Estado, tanto en un sentido interpersonal, como inter-territorial. Respecto a la redistribución territorial, el autor señala que con la reducción de las disparidades de ingreso interpersonal, no existe una corrección mecánica de las disparidades inter-territoriales, debido a que el hecho de que existan zonas de bajo ingreso, no solo se explica porque estén habitados por individuos de bajo ingreso, sino también debido a que las unidades territoriales son entidades socio-políticas diferenciadas, en las cuales existen distintos grados de infraestructura, concentración de actividad económica y oportunidades de trabajo (Prud'homme, 1995)

La literatura reconoce dos instrumentos concretos de política pública, que desde el enfoque de la descentralización fiscal apuntan a superar las disparidades regionales: los "Fondos de Compensación Regional" y los "Fondos de Desarrollo Territorial". Los fondos de compensación regional están ideados desde la perspectiva de la solidaridad territorial, buscan "financiar proyectos sociales y de infraestructura en aquellas regiones con niveles de pobreza por encima de la media nacional. De esta manera, los recursos estarían focalizados en regiones rezagadas" (Centro de Estudios Económicos Regionales, 2007). Las experiencias del FNDR en Chile y del Fondo de Compensación Inter-Regional Español, entre otros, muestran que esta "focalización" se logra a través del establecimiento de criterios económicos y socio-demográficos, establecidos por ley, para el reparto de los recursos. Es decir, buscan nivelar respecto a condiciones básicas de bienestar.

Los fondos de desarrollo territorial, por el contrario, están concebidos desde una perspectiva intersectorial y ponen atención en los recursos subutilizados de un territorio y sus posibles complementariedades, incluyendo usualmente a más de un territorio (Organización para la Cooperación y el Desarrollo Económico, 2009: 66-67). Los cambios en los fondos estructurales en la Unión Europea, es el principal ejemplo de Fondo de Desarrollo Territorial, en donde, no obstante existe una focalización geográfica, las unidades subnacionales cuentan con dos instrumentos: las "inversiones territoriales integrales", y los "Desarrollos Locales Liderados por la Comunidad" que permiten romper las lógicas sectoriales para hacer confluir distintas fuentes de financiamiento hacia iniciativas de relevancia para un territorio (Fresno, 2013). 


\section{Disparidades regionales y desarrollo territorial}

Las perspectivas del desarrollo territorial indican la íntima relación entre territorio- capital social competitividad. La competitividad de los distintos sectores productivos depende de cómo los distintos actores de los sectores productivos (empresas, universidades, comunidad, organizaciones sociales, gobierno) son capaces de cooperar para generar encadenamientos productivos, aprovechando sus ventajas competitivas (Silva, 2003). La capacidad asociativa depende del capital social de los actores, y la generación del capital social estaría fuertemente en lo territorial (Boiser, 1999). Este mismo autor identifica seis factores del desarrollo endógeno territorial: 1) Los actores presentes en el territorio, en relaciones de interdependencia y poder que son quienes, finalmente toman las decisiones o las influencian de algún modo; 2) Las instituciones, tanto públicas como privadas, presentes en el territorio, evaluadas según su velocidad de reacción, flexibilidad para responder a distintas situaciones, su virtualidad, es decir, penetración en los medios web y capacidad de aprendizaje; 3) La cultura, ya que determina los grados de confianza e identidad del territorio; 4) Los procedimientos administrativos del Estado, entre las que son de interés los instrumentos de fomento para el desarrollo, modelo de prestación de servicios del gobierno, y la forma en que se maneja la información; 5) Los Recursos, tanto materiales (Recursos Naturales y Físicos) como inmateriales (Recursos Humanos, Capital Social, Capital Cultural, Capital Cognitivo, etc) y; 6) El Entorno, definido principalmente por el Estado central, el mercado internacional y las relaciones horizontales con otros territorios (Boiser, 1997). Estas seis características no bastan para generar el proceso de desarrollo endógeno, sino que además deben estar adecuadamente articuladas, de forma densa y direccionada a través de un proyecto político.

Los gobiernos subnacionales pueden influir de distintas maneras en el desarrollo territorial de una región determinada, incidiendo en su competitividad, eficiencia en el uso de sus recursos naturales y socio-culturales y capacidad de captar recursos públicos y privados, entre las que destacan: a) Infraestructura de Calidad; b) Reglamentación que propenda a la eficiencia productivo-empresarial; c) Fomento a servicios fundamentales para el surgimiento de iniciativa privada; d) Fomento a la cooperación entre empresas; e) Provisión de servicios sociales según criterios territoriales (Elizalde, 2003). Sin embargo, como se ha dicho previamente, se requiere no sólo de iniciativas aisladas, sino además de una coherencia entre las distintas iniciativas llevadas a cabo por los gobiernos subnacionales. Es por ello que Boisier (1982) indica, a modo de propuesta teórica, que las principales decisiones (o grupos de decisiones) de actores tanto públicos como privados que inciden en las distintos factores del desarrollo territorial son: 1) La participación de las regiones en los ingresos nacionales; 2) El impacto regional de las políticas macroeconómicas y; 3) La capacidad de organización social del territorio.

Particularmente referido a la distribución de los ingresos del Estado por las distintas unidades territoriales, es decir a la función de asignación, Boiser (1982) señala que en países en que se privilegia el crecimiento económico agregado y la maximización del ritmo de crecimiento, se favorece la distribución de recursos en el "centro" nacional o metrópolis; y la desconcentración territorial es una tendencia que se utiliza debido a las externalidades negativas provocadas por la concentración excesiva que llevarían a desconcentrar parte del aparato productivo (Boiser, 1982, 1992). Así también, los recursos tienden a destinarse a los territorios prioritarios de desarrollo debido al más bajo costo de oportunidad del dinero, en donde se invertirá hasta cumplir con las "prioridades de desarrollo" que hayan sido definidas.

Así, existiría un tipo de planificación para las regiones centrales, caracterizado por el control/limitación sobre la actividad económica de la misma. Existiría otro tipo de planificación para las regiones prioritarias, de carácter activo, incentivando la iniciativa empresarial y ha generado las condiciones para la misma a través de la institucionalidad pública, existiría un tercer tipo de planificación para aquellos territorios que tienen algún grado de prioridad "residual", en el que esta sería de carácter innovador, pero modesta y limitada, mientras que en la mayoría de los territorios (aquellos sin prioridad) la planificación tendría como función la estabilización del ritmo de crecimiento mínimo (Boiser, 1982).

Existe una amplia literatura acerca de las disparidades territoriales en los grados de desarrollo y ritmos de crecimientos al interior de los países y regiones. Al analizarse las disparidades territoriales desde la perspectiva del modelo de crecimiento exógeno Solow-Swan, aplicado a nivel regional, se obtiene que la predicción de convergencia del ingreso entre territorios no es concluyente, sino que por el contrario, existiría un alto grado de convergencia en el ritmo de crecimiento entre las regiones de ingresos medios y altos, mientras existiría una heterogeneidad en los ritmos de crecimiento, con una tendencia al estancamiento, de las regiones caracterizadas como de "ingresos bajos" (Cuervo, 2003, 2006; Silva, 2003).

Desde la Nueva Geografía Económica, esto pue- 
de ser explicado por distintos factores de carácter económico, tales como la presencia de economías de escala, barreras en el comercio internacional, costos de transporte significativos, externalidades del mercado del trabajo, patrones de difusión del conocimiento, encadenamientos productivos, locación de materias primas, etc. Si bien en un principio, muchas ciudades son candidatas a concentrar un volumen importante de actividad económica "pequeñas diferencias (capricho histórico o expectativas autorrealizadas) determinan quienes son los ganadores y, una vez esto ha sido definido, opera un efecto inercial y acumulativo de aseguramiento de esta condición" (Cuervo, 2003: 70) generándose un patrón de dependencia histórico que refuerza la concentración en los centros económico-urbanos ya existentes, acumulando conocimiento, tradiciones, instituciones y poder político.

\section{Política local: participación y cliente- lismo}

Los procesos de descentralización no pueden ser pensados sólo desde la perspectiva económica, sino que también requiere que sean analizados dentro del proceso político de la región. Los gobiernos locales (sea a nivel comunal como regional) pueden ser conceptualizados como Interfaces socio-estatales, es decir espacios de intercambio, en el cual diversos actores se relacionan intencionadamente y ponen en juego sus diversos proyectos políticos, fuerzas y estrategias (Isunza, 2004). El grado de descentralización de un gobierno local muestra también, desde esta perspectiva, el grado de apertura del conflicto existente. La descentralización es una forma de institucionalizar el conflicto por el reparto de recursos en una esfera específica de la sociedad (un territorio) que permite darle gobernabilidad a la misma, tanto en su dimensión económica como política. La existencia o no de autoridades electas democráticamente resulta clave, ya que influye en el grado de legitimidad que tienen para poder mediar entre múltiples intereses dentro de un territorio y por tanto proveer dicha gobernabilidad.

Ahora bien, dentro de la dimensión política de la descentralización, la literatura admite la existencia tanto de elementos positivos como negativos asociados a esta. Desde la perspectiva de la gobernanza y la gestión de redes de política pública, la descentralización es una oportunidad para fomentar la participación ciudadana en la comunidad local, en lo que se ha denominado "gobernanza de proximidad" (Blanco y Gomá, 2003). En esta lógica, el resultado de la participación ciudadana es la emergencia de proyectos políticos locales (usando el lenguaje de Boisier) o "concertación local", es decir espacios en los cuales, a través de la participación directa de las organizaciones de base, existen prácticas de encuentro, negociación, deliberación y construcción de acuerdos (De la Maza, 2010).

Sin embargo, también se han destacado elementos negativos de la gestión local, en particular el problema del clientelismo político. Este puede ser definido como una forma de capital social, con características particulares: 1) El ser una relación asimétrica o inter-clase, pues entre personas con distintos niveles de capital económico y social, 2) Que opera según el principio de solidaridad difusa, y 3) Que se funda de manera mixta, tanto en factores simbólicos (ideología, lealtad, credibilidad) como por instrumentales (acceso a bienes públicos, recursos económicos o favores); 4) Opera tanto por mecanismos formales (servicios públicos y partidos políticos) como informales (juntas de vecinos, e incluso círculos de amigos) (Schröter, 2010; Durnston, 2005).

Existirían tres tipos fundamentales de relaciones de clientelismo político: 1) La relación patróncliente, tradicional en sectores tradicionales de la sociedad, basada en el intercambio personales interclase de bienes y servicios, en que la "clientela" está al arbitrio del "patrón" que cuenta con total autonomía; 2) Los "brokers" o "intermediarios", forma moderna de clientelismo, en la que existe este intermediario entre los "clientes" y el "caudillo", que pueden ser miembros de un partido político y organización social, organizándose redes, teniendo las organizaciones sociales y clientes mayor autonomía para cambiar de red clientelar; y 3) El semi-clientelismo, practicado por partidos políticos ?reformistas? que también incurren en intercambio clientelares, pero que también incluyen formas de inclusión y empoderamiento social no-clientelares (Durnston, 2005).

Las estrategias electorales de carácter personalistas requieren de una amplia base de apoyo al candidato en barrios y organizaciones de base en el territorio en el que compite, que normalmente se logran mediante intercambios de carácter clientelares (Miranda, 2008) particularmente en las formas de clientelismo moderno (Barozet, 2006) de allí que la esfera local es donde preferentemente se desarrollan las redes clientelares. Esto es particularmente relevante en Chile, en donde hay razones para creer que las redes clientelares a nivel local se han fortalecido en el Chile post-dictatorial producto de: 1) Un incremento del gasto público desconcentrado y/o descentraliado; 2) Un fortalecimiento del rol de los municipios en la asignación y provisión de servicios sociales; 3) la alta segmentación urbana en las comunas más pobladas (Barozet, 2008). 


\section{Metodología}

Este estudio se realizó siguiendo la metodología de "estudio de caso", a través del estudio de la asignación de FNDR de las regiones a las comunas en Chile, con múltiples unidades de análisis incrustadas (Yin, 2003), en este caso regiones del país, buscando determinar tendencias comunes en todos ellos, utilizando datos agregados que permitan establecer tendencias entre las distintas sub-unidades del caso. Es decir, sería un caso $(\mathrm{N}=1)$ que tendría quince sub-casos incrustados (embedded).

El diseño es de carácter explicativo y cuantitativo, en el que se mide, a través de métodos estadísticos, la influencia y peso específico de distintas variables en la asignación de los FNDR, existiendo una primera fase exploratoria en la que se analizan las principales características del proceso de FNDR en términos conceptuales y cuantitativos, y una segunda fase de carácter explicativa, en la que se utilizarán modelos econométricos para explicar la variable dependiente (FNDR comunal) por las diversas variables definidas como explicativas.

Respecto a la primera fase exploratoria del estudio, se utilizarán diversas fuentes de información, recopilando antecedentes a partir de entrevistas con actores dentro del proceso, revisión de antecedentes y documentos oficiales, revisión de la literatura académica pertinente, y el análisis de datos tanto internacionales como nacionales respecto a la inversión pública sub-nacional y el FNDR

Respecto a la fase explicativa del estudio, se utilizarán fuentes de información secundarias. De estas, las principales son los registros estadísticos de distintos servicios públicos del país, particularmente al Ministerio de Desarrollo Social, respecto de los proyectos de distintos tipos presentados y aprobados por cada municipio en diversos años, y en particular para los datos de los proyectos FNDR desglosado por año y comuna; la SUBDERE, respecto a la composición política de los consejos regionales; y el INE, para estimar la población del país en base a los CENSOS 1992, 2002 y 2012.

Dado el período para el que estaban disponibles los datos sobre la variable a explicar, (los montos de inversión) no fue posible contar con los PIB comunales, ya que el banco central no elabora dicha información, ni con los IDH comunales, ya que estos últimos solo se encuentran disponibles hasta el 2004; ni con el índice de pobreza comunal, ya que solo desde el 2009 incorpora la mayoría de comunas, siendo su número variable en cada encuesta CASEN, por lo que se utilizó como Proxy a dichas variables las categorías generadas por SUBDERE de desarrollo por comuna para el 2008. Respecto a la contabilización de los consejeros regionales por provincia y región se tuvo particularmente cuidado con: a) Los cambios provocados el 2008, con la generación de las regiones VIX y XV; b) Los cambios provocados por la creación de nuevas comunas, desde el 2000 y; c) Los cambios demográficos en las regiones XIII y $\mathrm{V}$, que con la misma fórmula cambiaron el prorrateo de los consejeros regionales entre provincias. Finalmente, respecto a los datos de composición de los consejeros regionales, sólo se obtuvo la información desde el año 2005, lo que implicará que los análisis para esta variable deban hacerse de forma separada y/o adicional al análisis general debido a la menor cantidad de períodos con los que cuenta.

Respecto a los datos de inversión pública extraídos por el SNI sobre los proyectos por comuna, es necesario hacer algunas observaciones adicionales. La base de datos original se limpió de tal manera de solo considerar aquellos proyectos que indicaban impacto por comuna, o recodificando dicha información en situaciones puntuales, de igual modo que todos aquellos casos con igual código de ingreso SNI, lo que indicaba que es el mismo proyecto sometido a revisión varias veces hasta que consiguiese la aprobación. Finalmente, se detectó que en los períodos 1994-1995-1996, la cantidad de proyectos ingresados al sistema es mucho menor que la cantidad de los años siguientes, en los que la inversión vía FNDR alcanza la gran mayoría de las comunas del país.

Finalmente, la cantidad de proyectos analizados fue de 40.851. Posteriormente se agregaron los datos en base a las comunas y los años, para obtener el FNDR para cada comuna en cada año, obtenido 5.391 casos de comuna/año con inversión FNDR para el período comprendido entre los años 1997 al 2012, lo que pudo corresponder a uno o más proyectos aprobados. Aquello requiere, por tanto, dos aclaraciones: a) puede existir un sesgo en los datos a subestimar la inversión en comunas de mayor población y/o desarrollo, pues muchas inversiones a pesar de estar localizadas en una zona específica, se las codificaba como de "carácter regional"; y b) los datos de FNDR a utilizar, serán siempre los basados en los 40.851 casos, por lo que a los resultados por comuna, región, o nacionales, se les agregará el epíteto "inversión comunal" distinguiendo de la cuenta que pudiese sacarse si se contabilizase las inversiones de impacto provincial y regional que no han sido recodificadas, así como las inversiones interregionales.

El método particular de análisis para los datos serán análisis estadístico de carácter cuantitativo, pues se busca determinar la relación de las distintas variables con los montos de inversión pública, 
Tabla 1: Operacionalización de Variables

\begin{tabular}{|c|c|c|}
\hline Dimensiones & Variables & Indicadores \\
\hline \multirow[t]{2}{*}{ Política } & \multirow{2}{*}{$\begin{array}{l}\text { Influencia } \\
\text { Partidaria }\end{array}$} & Coincidencia en la filiación partidaria entre alcaldes y CORES \\
\hline & & Coincidencia en la filiación partidaria entre alcaldes y gobiemo \\
\hline \multirow[t]{3}{*}{ Institucional } & \multirow{2}{*}{$\begin{array}{l}\text { Poder } \\
\text { Institucional }\end{array}$} & Comuna asiento de capital regional \\
\hline & & Comuna asiento de capital provincial \\
\hline & $\begin{array}{l}\text { Representación } \\
\text { Territorial }\end{array}$ & Cantidad de Consejeros Regionales por Comuna \\
\hline \multirow[t]{2}{*}{ Burocrática } & \multirow{2}{*}{$\begin{array}{l}\text { Capacidad } \\
\text { Organizativa }\end{array}$} & Cantidad de RS que obtiene una municipio al año \\
\hline & & $\%$ de RS que obtiene un municipio al año \\
\hline \multirow[t]{2}{*}{ Socio-Demográfica } & Población & Población Comumal \\
\hline & $\begin{array}{l}\text { Desarrollo } \\
\text { Comunal }\end{array}$ & Grado de desarrollo comunal según tipología SUBDERE \\
\hline
\end{tabular}

Fuente: Elaboración propia

sin embargo, habrá distintos métodos de análisis dependiendo del tipo de variable que se esté analizando: regresiones, análisis de factores y correlaciones bivariados. También es necesario hacer una segunda distinción, estos análisis -inicialmente- se realizarán con la totalidad de la información, pero una vez realizado esto, se procederá a realizar un análisis detallado por región, que permita determinar cómo los factores explicativos detectados varían de una región a otra, si es que lo hacen.

Para poder medir y relacionar las distintas variables se recurrió a los siguientes indicadores:

\section{Resultados}

Se buscó construir un modelo que explique la asignación de FNDR en las comunas, tanto a nivel nacional como por región. A continuación se adjunta el modelo con las cinco variables definidas en el apartado anterior, más la variable año de asignación, para el conjunto de la base de datos, es decir, a nivel nacional. Se realizarán dos regresiones ya que es necesario diferenciar el efecto que tiene el utilizar la variable "Municipio con Apoyo CORE" que sólo tiene valores desde el 2005.

A pesar de que todos los coeficientes del modelo son significativos al 0,05 ; la bondad de ajuste $\left(R^{2}\right)$ de ambos modelos es de 0,198 y 0,202. Es decir, el modelo solo explica en un $20 \%$ la variabilidad de la variable dependiente. Por ello se revisarán los modelos por Región, excluyendo las regiones XIV y XV debido a que no cuentan con la cantidad de observaciones necesarias para lograr una regresión suficientemente robusta.

A continuación se adjunta, de modo de lograr una visión general, pero diferenciada por región, dos cuadros resúmenes del resultado elaborado a partir de haber realizado el mismo procedimiento de re- gresión lineal, pero para las 13 regiones definidas anteriormente. La Tabla 14, muestra las bondades de ajuste de los modelos para las trece regiones definidas. Como se puede apreciar, estos cobran valores bastante distintos variando entre 0,339 para la Región del Bio Bio y 0,690 para la Región de Aysén para el caso de las regresiones 1997-2012.

Los $R^{2}$ de todas las regresiones para las trece regiones, pero desde 2005 al 2012 debido a que en este caso se incluye la variable "Municipio con Apoyo CORE" que solo tiene datos desde el 2005. En primer lugar, es necesario indicar que los $R^{2}$ de las regresiones de doce de las trece regiones aumentaron de forma significativa (entre 0,05 y 0,15 ). La excepción es la regresión de la región Metropolitana que disminuyó su bondad de ajuste hasta ser el más bajo $(0,273)$ mientras que la regresión de la región de Magallanes tiene el $R^{2}$ más alto $(0,843)$.

Las tablas 8 y 9 muestran la bondad de ajuste y los coeficientes respectivamente de las regresiones corregidas, es decir, introduciendo solo aquellas variables que aumentan de forma estadísticamente significativa el poder explicativo del modelo de regresión. Ante ello es necesario indicar que la variable "Municipio con Apoyo CORE" no fue estadísticamente significativa para ninguna de las trece regiones, y por tanto, no se requirió hacer regresiones en dos períodos temporales distintos, sino que sólo fueron realizadas en el período 1997-2012.

Comparando la Tabla 8 de los $R^{2}$ de los modelos corregidos con la Tabla 14 de los modelos iniciales, se puede indicar que los cambios en los $R^{2}$ son bajos, en relación con su valor, no sobrepasando el valor de 0,05. Los $R^{2}$ fluctúan entre los valores 0,337 y 0,687 lo que demuestra la gran variación existente entre regiones, tendencia que se corroborará y matizará al interpretar los coeficientes.

La Tabla 9 muestra los coeficientes de las correlaciones corregidas para los períodos 1997-2012 por 
Tabla 2: Bondad del Ajuste del Modelo 2005-2012 para Variable Inversión FNDR comunal

\begin{tabular}{|l|l|l|l|l|}
\hline Model & R & R Square & Adjusted R Square & Std. Error of the Estimate \\
\hline 1 &, $445^{a}$ &, 198 &, 197 & 7457305,239 \\
\hline
\end{tabular}

Fuente: Elaboración propia

Tabla 3: Coeficientes del Modelo 2005-2012 para Variable Inversión FNDR comunal y su Significancia

\begin{tabular}{|c|c|c|c|c|c|c|}
\hline \multirow{2}{*}{\multicolumn{2}{|c|}{ Model }} & \multicolumn{2}{|c|}{ Unstandardized Coefficient5 } & \multirow{2}{*}{\begin{tabular}{|l|} 
Standardized Coefficients \\
Beta
\end{tabular}} & \multirow[b]{2}{*}{$t$} & \multirow[b]{2}{*}{ Sig. } \\
\hline & & B & Std. Error & & & \\
\hline \multirow[t]{7}{*}{1} & (Constant) & $-6,825 \mathrm{E} 8$ & $1,287 \mathrm{E} 8$ & & $-5,303$ &, 000 \\
\hline & Población Comunal & 56,497 & 2,596 &, 487 & 21,767 &, 000 \\
\hline & $\begin{array}{l}\text { Porcentaje del CORE que tiene una } \\
\text { Provincia en relación al total regional }\end{array}$ & 372219,544 & 395739,848 &, 016 & 941 &, 347 \\
\hline & Municipio con Apoyo CORE & 564346,903 & 314828,118 &, 034 & 1,793 &, 073 \\
\hline & Municipio con Apoyo Gobiemo & $-589195,008$ & 313112,671 &,- 035 & $-1,882$ &, 060 \\
\hline & Grado de Desarrollo Comunal & 584490,198 & 136794,137 &, 096 & 4,273 &, 000 \\
\hline & Año de Asignación & 339577,524 & 64073,278 &, 093 & 5,300 &, 000 \\
\hline
\end{tabular}

a. Dependent Variable: Costos Agregados de los proyectos asignados por año en miles de pesos

Fuente: Elaboración propia

Tabla 4: Bondad del Ajuste del Modelo 1997-2012 para Variable Inversión FNDR comunal

\begin{tabular}{|l|l|l|l|l|}
\hline Model & R & R Square & Adjusted R Square & Std. Error of the Estimate \\
\hline 1 &, $450^{\mathrm{a}}$ &, 202 &, 201 & 5677812,394 \\
\hline
\end{tabular}

Fuente: Elaboración propia

Tabla 5: Coeficientes del Modelo 1997-2012 para Variable Inversión FNDR comunal y su Significancia

\begin{tabular}{|c|c|c|c|c|c|}
\hline \multirow[b]{2}{*}{ Model } & \multicolumn{2}{|c|}{ Unstandardized Coefficients } & \multirow{2}{*}{$\begin{array}{l}\text { Standardized Coefficients } \\
\text { Beta }\end{array}$} & \multirow[b]{2}{*}{$\mathrm{t}$} & \multirow[b]{2}{*}{ Sig. } \\
\hline & $\mathrm{B}$ & Std. Error & & & \\
\hline 1 (Constant) & $-6,687 \mathrm{E} 8$ & $3,403 \mathrm{E} 7$ & & $-19,653$ &, 000 \\
\hline Población Comunal & 39,879 & 1,491 &, 428 & 26,742 &, 000 \\
\hline $\begin{array}{l}\text { Porcentaje del CORE que tiene una Provincia } \\
\text { en relación al total regional }\end{array}$ & 306796,205 & 258678,839 &, 015 & 1,186 &, 236 \\
\hline Municipio con Apoyo Gobiemo & $-92422,894$ & 156088,936 &,- 007 &,- 592 &, 554 \\
\hline Grado de Desarrollo Comunal & 504545,742 & 74450,308 &, 108 & 6,777 &, 000 \\
\hline Año de Asignación & 333207,494 & 16978,133 &, 241 & 19,626 &, 000 \\
\hline
\end{tabular}

a. Dependent Variable: Costos Agregados de los proyectos asignados por año en miles de pesos

Fuente: Elaboración propia 
Tabla 6: Bondad del Ajuste de las Regresiones 1997-2012 para Variable Inversión FNDR comunal, por Región

\begin{tabular}{|l|l|l|l|l|}
\hline Región del Pais & R & R Square & Adjusted R Square & Std. Error of the Estimate \\
\hline Tarapaca &, $747^{\mathrm{c}}$ &, 557 &, 542 & $3,456 \mathrm{E}+06$ \\
\hline Antofagasta &, $807^{\mathrm{b}}$ &, 651 &, 638 & $1,414 \mathrm{E}+07$ \\
\hline Atacama &, $829^{\mathrm{c}}$ &, 688 &, 676 & $4,237 \mathrm{E}+06$ \\
\hline Coquimbo &, $815^{\mathrm{c}}$ &, 664 &, 657 & $4,851 \mathrm{E}+06$ \\
\hline Valparaígo &, $802^{\mathrm{d}}$ &, 643 &, 640 & $1,357 \mathrm{E}+06$ \\
\hline Ohiggins &, $598^{\mathrm{c}}$ &, 358 &, 352 & $1,918 \mathrm{E}+06$ \\
\hline Maule &, $684^{\mathrm{c}}$ &, 467 &, 461 & $3,249 \mathrm{E}+06$ \\
\hline Bio Bio &, $582^{\mathrm{d}}$ &, 339 &, 335 & $2,698 \mathrm{E}+06$ \\
\hline Araucania &, $656^{\mathrm{c}}$ &, 431 &, 425 & $2,748 \mathrm{E}+06$ \\
\hline Los Lagos &, $720^{\mathrm{c}}$ &, 519 &, 515 & $2,045 \mathrm{E}+06$ \\
\hline Aysén &, $830^{\mathrm{c}}$ &, 690 &, 680 & $1,876 \mathrm{E}+06$ \\
\hline Magallanes &, $806^{\mathrm{I}}$ &, 649 &, 637 & $9,633 \mathrm{E}+06$ \\
\hline Metropolitana &, $650^{5}$ &, 422 &, 419 & $2,118 \mathrm{E}+06$ \\
\hline
\end{tabular}

Fuente: Elaboración propia

Tabla 7: Bondad del Ajuste de las Regresiones 2005-2012 para Variable Inversión FNDR comunal, por Región

\begin{tabular}{|l|l|l|l|l|}
\hline Región del Pais & R & R Square & Adjusted R Square & Std. Error of the Estimate \\
\hline Tarapacá &, $858^{\mathrm{a}}$ &, 735 &, 709 & $3,716 \mathrm{E}+06$ \\
\hline Antofagasta &, $848^{\mathrm{b}}$ &, 719 &, 693 & $1,675 \mathrm{E}+07$ \\
\hline Atacama &, $854^{\mathrm{c}}$ &, 729 &, 703 & $5,145 \mathrm{E}+06$ \\
\hline Coquimbo &, $901^{\mathrm{d}}$ &, 812 &, 802 & $4,482 \mathrm{E}+06$ \\
\hline Valparaíso &, $795^{\mathrm{e}}$ &, 632 &, 625 & $1,653 \mathrm{E}+06$ \\
\hline O'Higgins &, $606^{\mathrm{I}}$ &, 367 &, 352 & $2,261 \mathrm{E}+06$ \\
\hline Maule &, $784^{\mathrm{s}}$ &, 615 &, 605 & $3,645 \mathrm{E}+06$ \\
\hline Bí-Bio &, $629^{\mathrm{d}}$ &, 396 &, 387 & $3,277 \mathrm{E}+06$ \\
\hline Araucania &, $830^{5}$ &, 690 &, 682 & $2,482 \mathrm{E}+06$ \\
\hline Los Lagos &, $730^{\mathrm{a}}$ &, 532 &, 522 & $2,526 \mathrm{E}+06$ \\
\hline Aysén &, $891^{\mathrm{e}}$ &, 795 &, 778 & $1,893 \mathrm{E}+06$ \\
\hline Magallanes &, $918^{\mathrm{s}}$ &, 843 &, 829 & $8,953 \mathrm{E}+06$ \\
\hline Metropolitana &, $522^{\mathrm{L}}$ &, 273 &, 261 & $2,691 \mathrm{E}+06$ \\
\hline
\end{tabular}

Fuente: Elaboración propia 
Tabla 8: Bondad del Ajuste de las Regresiones Corregidas 1997-2012 para Variable Inversión FNDR comunal, por Región

\begin{tabular}{|l|l|l|l|l|}
\hline Región del Pais & R & R Square & Adjusted R Square & Std. Error of the Estimate \\
\hline Tarapacá &, $739^{a}$ &, 545 &, 536 & 3395993,026 \\
\hline Antofagasta &, $798^{a}$ &, 638 &, 632 & $1,426 \mathrm{E}+07$ \\
\hline Atacama &, $829^{4}$ &, 687 &, 678 & 4229386,941 \\
\hline Coquimbo &, $808^{a}$ &, 652 &, 650 & 4903279,636 \\
\hline Valparaiso &, $801^{a}$ &, 642 &, 641 & 1356574,497 \\
\hline O'Higgin &, $598^{4}$ &, 358 &, 353 & 1916630,243 \\
\hline Maule &, $680^{4}$ &, 462 &, 459 & 3252862,903 \\
\hline Bio-Bio &, $581^{a}$ &, 337 &, 335 & 2690254,415 \\
\hline Araucania &, $654^{a}$ &, 428 &, 425 & 2733958,493 \\
\hline Los Lagos &, $719^{4}$ &, 517 &, 514 & 2046357,006 \\
\hline Aysén &, $828^{a}$ &, 686 &, 682 & 1870309,381 \\
\hline Magallanes &, $799^{4}$ &, 638 &, 633 & 9688301,120 \\
\hline Metropolitana &, $644^{a}$ &, 415 &, 414 & 2134976,744 \\
\hline
\end{tabular}

Fuente: Elaboración propia

región y su significancia. En base a ello, se pueden rescatar tres regularidades empíricas válidas para todas o casi todas las regiones: a) En las regresiones de todos los modelos, el coeficiente de la variable "año de asignación" es significativo, con una relación positiva, aquello indica estadísticamente, en términos reales (pues los datos de inversión están normalizados al 2012) hubo crecimiento de la inversión vía FNDR de carácter comunal; b) En las regresiones de todos los modelos el coeficiente de la variable "Población Comunal" es significativo, pero no solo eso, sino que salvo en la Región Metropolitana $(\mathrm{RM})$ fue el coeficiente que en términos estandarizados, tiene un mayor peso en el poder explicativo del modelo; c) Tal como se mencionó anteriormente para las regresiones de todas las regiones, los coeficientes de las variable "Municipio con Apoyo CORE" no fueron estadísticamente significativos.

Vinculado con lo anterior, particularmente con el punto "b", es que al comparar las regresiones por región, de las trece regresiones, en las correspondientes a la Región de Antofagasta, Región de Coquimbo, Región del Bio Bio, Región de Aysén, Región de Magallanes y Región Metropolitana, solo se introdujo la variable población comunal y año de asignación, teniendo $R^{2}$ bastante significativos en algunos casos, lo que refuerza la idea de que la variable población comunal es aquella que en todos los modelos tiene un mayor peso explicativo. Las variables "Grado de Desarrollo Comunal" y "Porcentaje del CORE Regional elegido por provincia" fueron aquellas incluidas, por si solas o incluso ambas a la vez, en los modelos de las otras regiones no señaladas en el párrafo anterior, teniendo una relación positiva con la variable dependiente, mientras que la variable "Municipio con Apoyo del Gobierno" apenas fue incluida en el modelo de regresión de una sola región, el de la Región de Tarapacá, teniendo además, el coeficiente con menor peso explicativo en dicho modelo.

En síntesis, se puede afirmar que existen tres grupos de regiones, aquellas en que la variable "Porcentaje del CORE Regional Elegido por Provincia" es significativo para explicar la variable inversión FNDR comunal (Región de Tarapacá, Región del Maule y Región de O?Higgins) aquellas en que la variable "Grado de Desarrollo Comunal" es significativa para explicar la variable inversión FNDR comunal (Región de Atacama, Región de Valparaíso, Región de la Araucanía y Región de los Lagos) y aquellas en que ninguna de estas variables es significativa (Región de Antofagasta, Región de Coquimbo, Región del Bío-Bío, Región de Aysén, Región de Magallanes y Región Metropolitana) que como se puede observar es el grupo con más regiones

Siendo estos los resultados generales, es decir, que la principal variable que explica la inversión FNDR comunal, y que en mucho menor grado y solamente en algunas regiones es explicada por las variables de carácter social, política y/o institucionales, cabe la pregunta ¿Es que acaso la inversión regional es una simple función de la población que habita en las comunas? ¿Acaso los datos de inversión FNDR comunal esconden las tendencias per cápita de las comunas? En ese sentido, una hipótesis factible es que si bien la variable inversión FNDR comunal mostró una relación baja o nula con las variables de carácter social, política y/o institucionales, aquello se podría deber a que se consideraron los montos en bruto, y que al considerar la variable en términos per cápita se podrían visibilizar algunas relaciones adicionales, particularmente con las variables políticas e institucionales. 
Tabla 9: Coeficientes de las Correlaciones Corregidas 1997-2012 para Variable Inversión FNDR comunal, Por Región y su Significancia

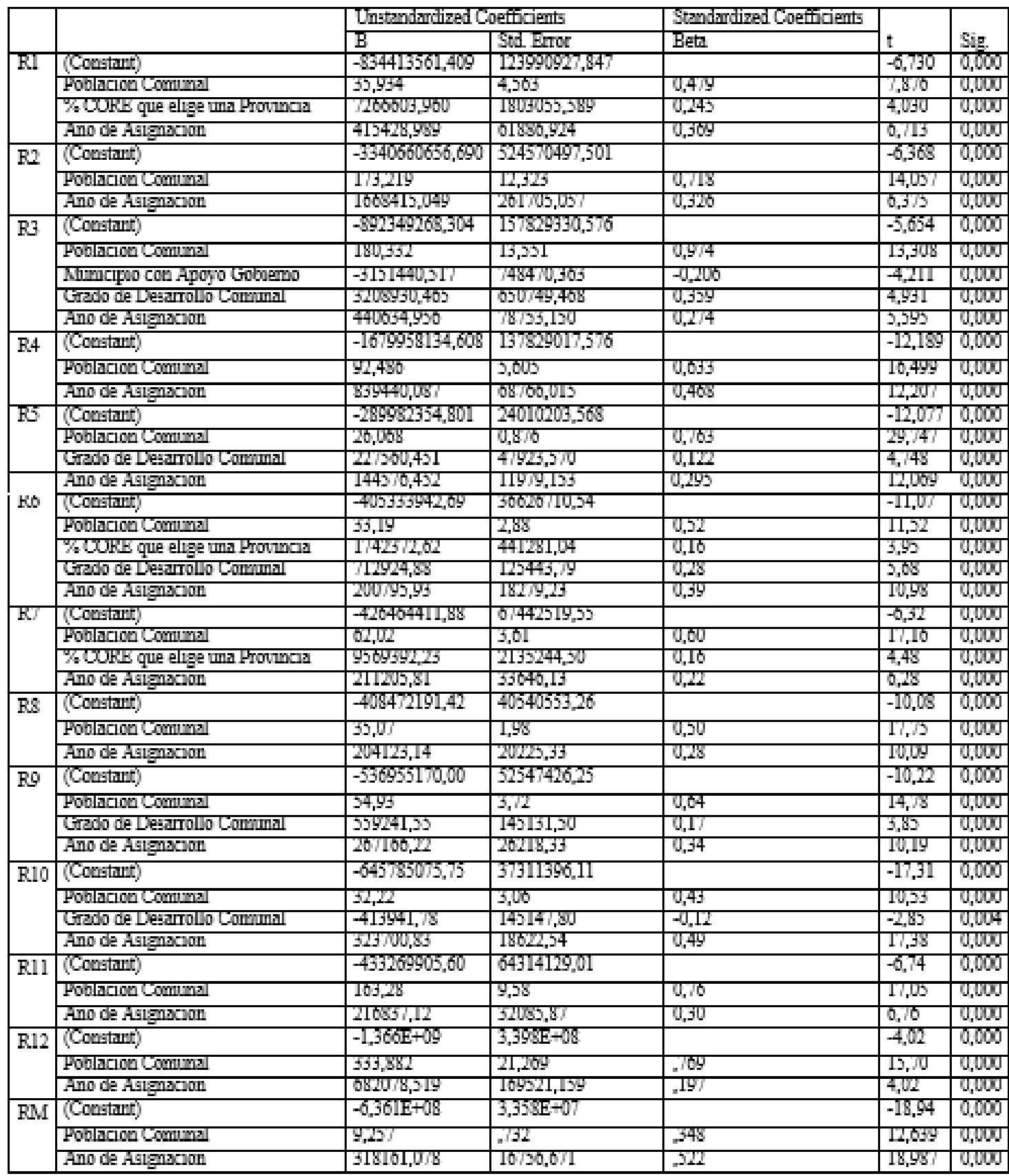

Fuente: Elaboración propia 
Por ello se procederá a realizar un análisis de regresión lineal para las trece regiones, pero ahora con la variable "Inversión FNDR comunal per cápita" como variable dependiente, y con las mismas variables independientes utilizadas para analizar la variable en forma bruta. Es necesario agregar, que debido a la distribución que toma la variable "Inversión FNDR comunal per cápita" se utilizó en forma logarítmica. Aquello afecta la interpretación de los resultados, que no es de forma lineal, sino que cada unidad de las variables independientes afecta en términos porcentuales promedios a la variable dependiente.

En la Tabla 10 se pueden observar los $R^{2}$ de las regresiones aplicadas para cada región para la variable dependiente en términos per cápita. Los valores de $R^{2}$ varían significativamente entre las regresiones de una región y otra, estando los valores entre 0,247 (Región del Maule) y 0,641 (Región de Coquimbo), lo que nos indica que las regresiones solo son modelos útiles para pronosticar el comportamiento de la variable dependiente en ciertas regiones, mientras que en otras, las variables explicativas no alcanzaron a explicar el $40 \%$ de la variabilidad de la variable dependiente (Región de Atacama, Región de Valparaíso, Región de O?Higgins Región del Maule, Región del Bío-Bío y Región de los Lagos) y por tanto, las regresiones para dichas regiones no pueden seguir siendo utilizadas ya que la asociación entre las variables independientes y la variable dependiente no es lo suficientemente fuerte.

Además, es necesario considerar que, al igual que para el ejercicio con la variable en términos brutos, no todos los coeficientes de las regresiones son estadísticamente significativos, lo que significa que es necesario retirar algunas variables. Esto debe realizarse región por región, ya que las regresiones de distintas regiones tienen como coeficientes estadísticamente significativos a aquellos de distintas variables. El retiro de algunas variables a las regresiones puede afectar los $R^{2}$, ya que en la medida de que algunas variables dependientes con menor peso explicativo son retiradas del modelo, también se pierde algún grado ajuste del mismo, aunque sea marginal.

La Tabla 12 muestra los $R^{2}$ de las Regresiones Corregidas para la variable Inversión FNDR comunal per cápita para las regiones en que el $R^{2}$ fue superior a 0,4. Como era de esperarse los valores $R^{2}$ variaron en cada una de las 7 regiones en que se realizó la nueva regresión, pero en un rango relativamente limitado, entre 0,46 (Región Metropolitana) y 0,6 (Región de Coquimbo)

La Tabla 13 muestra los coeficientes de las correlaciones corregidas para el período 1997-2012 para variable Log-Natural de la Inversión FNDR comunal per cápita, en las siete regiones en que el $R^{2}$ fue superior a 0,4. Para las siete regresiones realizadas, se pueden enunciar de forma general dos afirmaciones: 1) Que en las regresiones para las siete regiones, en todas ellas el "año de asignación" fue una variable significativa, aquello quiere decir que, consistentemente con los resultados de las regresiones con la variable "Monto FNDR comunal" en bruto, existe evidencia estadística para afirmar que, al menos en estas siete regiones, el monto FNDR per cápita comunal ha aumentado en términos reales en el tiempo incluso por encima del crecimiento de la población en las comunas; 2) Que nuevamente las variables de carácter político "Municipio con Apoyo CORE" y "Municipio con Apoyo del Gobierno" resultaron no significativas.

Las distintas regresiones por región para la variable "Log-Natural del FNDR comunal per cápita", al igual que con la variable en bruto, muestran que la variable dependiente es afectada de forma estadísticamente significativa por distintas variables y con distintos pesos explicativos por cada región. Como se observa en la Tabla 20, excepto por las regresiones para las Regiones de la Araucanía y Aysén, todas regresiones entre sí tienen combinaciones distintas de las tres variables independientes consideradas, lo que hace imposible algún tipo de clasificación de forma rigurosa.

Las variables que las regresiones regionales incorporan son "Porcentaje del CORE elegido por Provincia" (Región de Antofagasta, Región de la Araucanía, Región de Aysén y Región de Magallanes) "Población Comunal" (Región de Antofagasta, Región de Coquimbo, Región de Magallanes y Región Metropolitana) y "Grado de Desarrollo Regional" (Región de Tarapacá, Región de la Araucanía, Región de Aysén, Región de Magallanes y Región Metropolitana). Cabe destacar que comparando las variables incluidas en las regresiones con la variable dependiente per cápita y en términos brutos, en la mayoría de los casos cambiaron, lo que implica que el medir la variable dependiente en términos brutos y per-cápita, se está aludiendo efectivamente a dinámicas distintas, tal como se asume en la sección metodológica del estudio.

En todas las regresiones de la Tabla 20, en que se incluye la variable "Grado de Desarrollo Comunal" este tiene una relación directa (positiva) con la variable explicada, y recordando que la codificación de la variable indicaba que la categoría 1 era la de las comunas con mayor desarrollo y la categoría 5 de las comunas con menor desarrollo, se puede decir que para las regiones de Tarapacá, la Araucanía, Aysén, Magallanes y Metropolitana, que en promedio cuando una comuna tiene un nivel de desarrollo 
Tabla 10: Bondad del Ajuste de las Regresiones 1997-2012 por Región para Log-Natural Inversión FNDR comunal per cápita

\begin{tabular}{|c|c|c|c|c|c|}
\hline Region del Pai: & Mode & $\mathrm{R}$ & R Square & Adjusted R Square & Std. Brror of the Estimate \\
\hline Tarapaca & 1 & .725 & .526 & .507 & 1,27081 \\
\hline Antofagasta & 1 & .727 & .529 & .512 & 94523 \\
\hline Atacama & 1 &., 572 & 327 & 302 & .95257 \\
\hline Coquimbo & l & .801 & .641 & .633 & .64458 \\
\hline Valparaiso & 1 &., 559 & 312 & 306 & 91919 \\
\hline O'Higgins & l & .499 & 249 & 242 & 1,22125 \\
\hline Maule & 1 & .497 & 247 & 238 & 1,48722 \\
\hline Bio-Bio & 1 & .530 & 281 & 277 & 1,02956 \\
\hline Areucania & 1 & .648 & .420 & .414 & 84461 \\
\hline Los Lagos & 1 & .616 & 379 & 374 & 1,26274 \\
\hline Aysen & 1 &, 698 & .488 & .471 & .69721 \\
\hline Magallanes & 1 & .768 & .590 & .575 & 1,02503 \\
\hline Metropolitama & l &, 683 & .467 & .463 & 1,05903 \\
\hline
\end{tabular}

Fuente: Elaboración propia

Tabla 11: Bondad del Ajuste de las Regresiones Corregidas 1997-2012 en siete Regiones para Log-Natural Inversión FNDR comunal per cápita

\begin{tabular}{|c|c|c|c|c|}
\hline Regon del Pais & $\mathrm{R}$ & RSqure & Adjusted R Square & Std Enor of the Estimate \\
\hline $\mathrm{RI}$ & $.730^{2}$ & 533 & 526 & 1,2704 \\
\hline $\mathrm{B2}$ & $.701^{2}$ & 492 & $480^{\circ}$ & 97516 \\
\hline R4 & $777^{2}$ & 603 & 600 & 67354 \\
\hline RO & $659^{2}$ & 408 & 404 & 86377 \\
\hline RII & $690^{2}$ & 476 & 466 & 70038 \\
\hline $\mathrm{R} 2$ & $.765^{2}$ & 585 & 573 & 1,0257 \\
\hline $\mathrm{HW}$ & . $68 \mathrm{I}^{\mathrm{T}}$ & 464 & 462 & 1,06087 \\
\hline
\end{tabular}

Fuente: Elaboración propia 
Tabla 12: Coeficientes de las Correlaciones Corregidas 1997-2012 para Variable Log-Natural Inversión FNDR comunal per cápita, en Siete Regiones y su Significancia

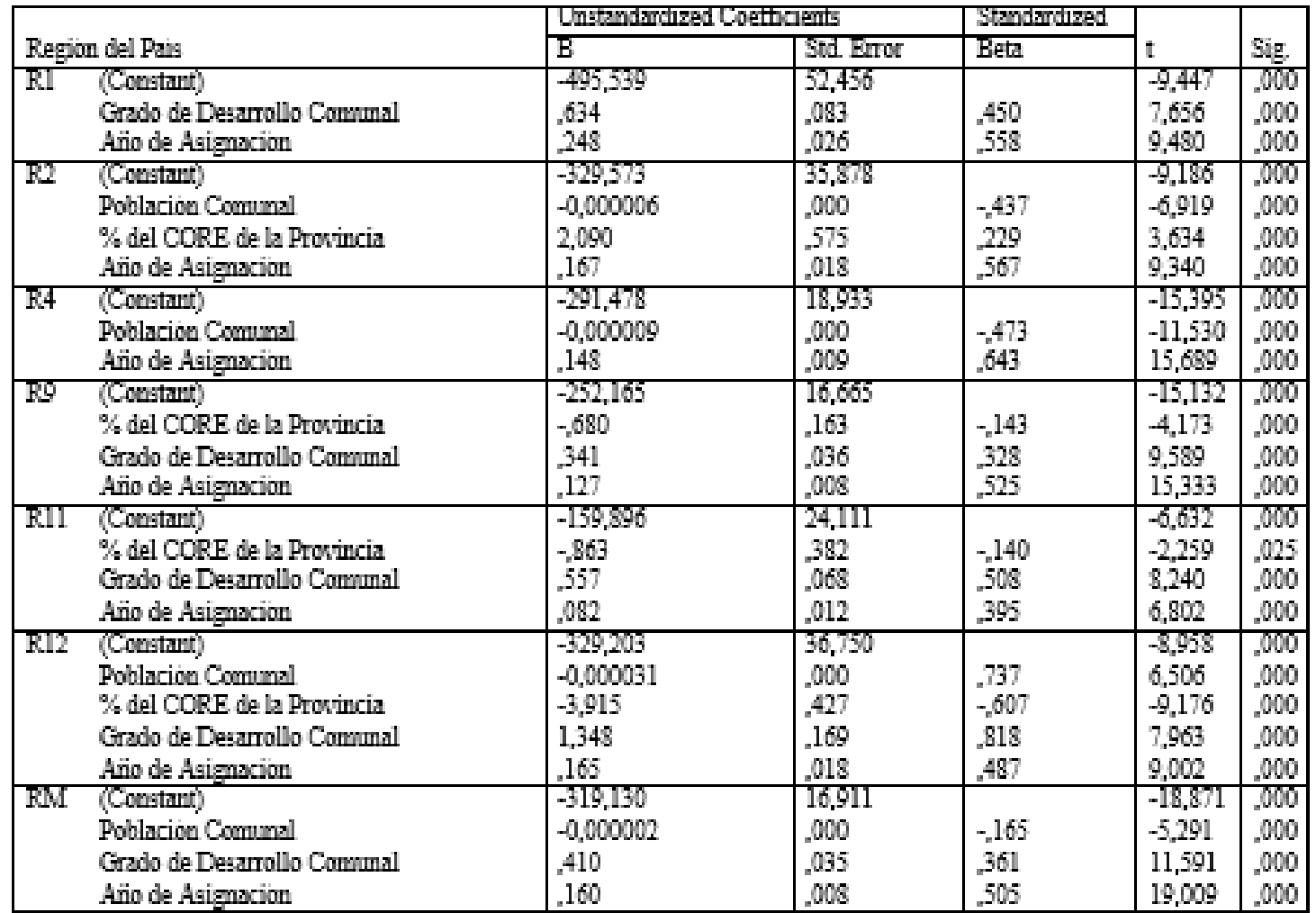

Fuente: Elaboración propia

menor en el Grado de Desarrollo Comunal, implica que aumenta porcentualmente su asignación FNDR per cápita.

Así mismo, en todas las regresiones de la Tabla 20, en que se incluye la variable "Población Comunal" esta tiene una relación inversa (negativa) con la variable explicada, por lo que se puede decir para las regiones de Antofagasta, de Coquimbo, de Magallanes y Metropolitana, que en promedio cuando una comuna tiene mayor población, implica que disminuye porcentualmente su asignación FNDR per cápita.

Para al caso de la variable introducida, "Porcentaje del CORE elegido por Provincia" en la regresión para la Región de Antofagasta se dio la relación en un sentido directo (positivo) como era de esperarse, es decir, una mayor cantidad de consejeros regionales en la provincia, implicaría un mayor poder de las comunas de dicha provincia en el CORE y por tanto mayor captación de recursos. Sin embargo, para las regiones de la Araucanía, de Aysén y de Magallanes, la relación se dio en un sentido inverso (negativo). Es decir, que en la medida que se tiene una mayor cantidad de consejeros regionales en la provincia, las comunas en dicha provincia tendrían en promedio, una disminución porcentual en su asignación de FNDR comunal en términos per-cápita. Aquello claramente es contra-intuitivo, sin embargo, puede ser explicado por la existencia, en los CORE de dichas regiones, de alguna regla o mecanismo compensatorio hacia ciertas comunas en base a criterios no considerados en el presente estudio.

Para efectos de simplificar la lectura de los datos, la Tabla 21 es un resumen de la evidencia estadística existente para las siete regiones en que se pudo realizar los últimos análisis de las variables que están relacionadas con la variable dependiente en términos per cápita y logarítmicos. La tabla muestra que la variable de control "año de asignación" está relacionada con la variable dependiente en las siete regiones, lo que constata el crecimiento real del FNDR en el tiempo. Las variables "grado de desarrollo comunal" estaría relacionada en cinco regiones con el monto de FNDR per cápita, y las variables "Porcentaje del CORE que elija la provincia" y "Población Comunal" estarían relacionadas en cuatro regiones con la variable dependiente, sólo existiendo dos regiones (las regiones de la Araucanía y de Aysén) con las mismas variables como relacionadas con el monto FNDR per cápita por comuna. 
Tabla 13: Resumen de las Variables Relacionadas con el Log-Natural Inversión FNDR comunal per cápita para cada región

\begin{tabular}{|l|l|l|l|l|l|l|l|l|}
\hline \multirow{2}{*}{ Variable } & \multicolumn{2}{|l|}{ Regiones } \\
\cline { 2 - 10 } & $\mathrm{R} 1$ & $\mathrm{R} 2$ & $\mathrm{R} 4$ & $\mathrm{R} 9$ & $\mathrm{R} 11$ & $\mathrm{R} 12$ & $\mathrm{RM}$ & Total \\
\hline Población Comunal & & $\mathrm{X}$ & $\mathrm{x}$ & & & $\mathrm{x}$ & $\mathrm{x}$ & 3 \\
\hline \% del CORE de la Provincia & & $\mathrm{x}$ & & $\mathrm{x}$ & $\mathrm{x}$ & $\mathrm{x}$ & & 4 \\
\hline Grado de Desarrollo Comunal & $\mathrm{x}$ & & & $\mathrm{x}$ & $\mathrm{x}$ & $\mathrm{x}$ & $\mathrm{x}$ & 5 \\
\hline Año de Asignación & $\mathrm{x}$ & $\mathrm{x}$ & $\mathrm{x}$ & $\mathrm{x}$ & $\mathrm{x}$ & $\mathrm{x}$ & $\mathrm{x}$ & 7 \\
\hline
\end{tabular}

Fuente: Elaboración propia

\section{Conclusiones}

Los resultados de los análisis realizados corroboran la pertinencia del enfoque intra-regional adoptado, toda vez que: a) En todas las regresiones realizadas, al utilizar los datos nacionales, los resultados fueron no significativos para todas las variables hasta el punto en que dichos análisis no brindaron ningún grado de información relevante. Sin embargo, cuando se hicieron los mismo análisis diferenciando por región, la significancia de los modelos $\left(R^{2}\right)$ aumentó de forma considerable y se pudo trabajar con dichos resultados; b) Para los modelos por región que utilizaron como variable dependiente, tanto la inversión FNDR comunal en términos brutos como per cápita aplicando logaritmo natural, hubo diferencias notorias entre regiones, tanto en la bondad del ajuste de los modelos, como en las variables que resultaron significativos en cada uno de ellos.

Lo anterior es muy importante, porque reconocer que los modelos basados en evidencia empírica que describen el comportamiento de las variables asociadas al FNDR, entendido como instrumento de descentralización fiscal, difieren significativamente de región en región, implica cuestionar la forma de aproximación al análisis de estos instrumentos ya clásica en el discurso de las políticas públicas en Chile, a saber, desde el diseño institucional de los procedimientos e incentivos que rigen a las organizaciones públicas e instrumentos de gestión de forma nacional, que al analizarse desde una perspectiva normativo-abstracta, necesariamente no dan cuenta de las diferencias entre regiones.

Respecto al tipo de análisis realizados, es necesario realizar una distinción entre los análisis con la variable dependiente en términos brutos y per cápita, que se expresó con cada vez más claridad en la medida en que se fueron realizando mayor cantidad de pruebas y análisis. Intuitivamente, basados en las metodologías oficiales del sistema nacional de inversión pública podría pensarse que la asignación de recursos reproduce de forma más o menos aséptica la distribución comunal de la población, o incluso la reforzaría, lo que sería apoyado por la evidencia empírica de las regresiones realizadas con la variable dependiente "Inversión FNDR comunal".

Si bien es cierto que, efectivamente, en la medida en que una comuna tiene más población tiene mayor inversión comunal FNDR en términos brutos, al observar las regresiones realizadas con la variable dependiente "Log-Natural de la Inversión FNDR comunal per cápita" lo que se observa es que en términos per cápita, cada región utiliza criterios distintos en el reparto de los recursos, de carácter socioeconómicos, de equidad territorial o incluso otros no considerados en el presente estudio, tanto por la relación inversa de la variable "Porcentaje del CORE elegido por la Provincia", como por las seis regresiones para seis regiones que ni siquiera alcanzaron un $R^{2}$ de 0,4 .

Las regresiones utilizando la variable dependiente "Inversión FNDR comunal" permiten analizar la inversión del FNDR desde la perspectiva de la "igualdad/desigualdad territorial", es decir, determinar, independientemente del tamaño o la población que habita en una comuna, cuales son los factores que inciden en que una comuna reciba una mayor o menor inversión de FNDR. Es decir, considerando la comuna como una unidad abstracta. En cambio las regresiones utilizando la variable dependiente "LogNatural de la Inversión FNDR comunal per cápita" permiten analizar la inversión del FNDR desde la perspectiva de la "equidad/inequidad territorial", es decir considerando las comunas no simplemente como unidades administrativas, sino como comunidades humanas sobre las que los recursos son distribuidos en mayor o menor medida.

Específicamente para las regresiones "Inversión FNDR comunal" en todas las regiones analizadas, se encontró evidencia significativa de que la variable que explica de mejor manera la distribución intraregional del FNDR es la población de las mismas, estando relacionada de manera directa con la variable dependiente. Dicho de otro modo, en la medida de que en una región, una comuna es más poblada, en promedio recibirá mayores recursos vía FNDR. Sin embargo, no queda claro si esto se debe a una 
mayor competitividad de las comunas con mayor población, o simplemente a un sesgo de las metodologías de análisis de tipo "costo-beneficio"

Además existe evidencia estadística de que en la Región de Tarapacá, Región del Maule y Región de O?higgins, en la medida en que una provincia elige a una mayor cantidad de consejeros regionales, las comunas de dicha provincia obtienen en promedio mayores recursos vía FNDR. Así mismo, para la Región de Atacama, Región de Valparaíso y Región de la Araucanía, se puede afirmar que en la medida en que una comuna tiene un menor grado de desarrollo, esta recibe mayor aportes vía FNDR, sin embargo, para la Región de los Lagos está relación es inversa.

Es necesario destacar que para las regresiones de las trece regiones analizadas, la variable independiente con mayor poder explicativo es la población comunal, muy por encima de las variables anteriormente expuestas.

Para las regresiones que utilizaron como variable dependiente "Log-Natural de la Inversión FNDR comunal per cápita" en cambio la situación es mucho más diversa de Región en Región. En primer lugar, porque para seis de las trece regiones analizadas no fue posible desarrollar, en base a la variables explicativas disponibles y definidas, un modelo de regresión logarítmico-lineal, que explicara de forma satisfactoria $\left(R^{2}>0,4\right)$ el comportamiento de la variable dependiente. Así pues, una de las principales conclusiones que se derivan de estos modelos es que para la Región de Atacama, Región de Valparaíso, Región del Maule, Región de O?Higgins, Región del Bío-Bío y Región de los Lagos no fue posible determinar los factores que determinan la distribución comunal del FNDR en términos per cápita.

Del total de siete regiones para las que sí fue posible determinar un modelo de regresión que explicase el comportamiento de la variable "Log-Natural de la Inversión FNDR comunal per cápita", en las Región de Antofagasta, Región de Coquimbo, Región de Magallanes y Región Metropolitana, se presenta una relación entre la variable dependiente y la variable población, pero a diferencia de cuando se utilizó la variable dependiente de forma bruta, para estos casos la relación es inversa. Aquello quiere decir que para cada una de estas cuatro regiones, en la medida de que una comuna dentro de las mismas tiene una población mayor, su asignación e inversión FNDR en términos per cápita será, en promedio, menor.

Por otro lado, en las regresiones con la variable "Log-Natural de la Inversión FNDR comunal per cápita" correspondientes a la Región de Tarapacá,
Región de la Araucanía, Región de Aysén, Región de Magallanes y Región Metropolitana, se encontró una relación directa (positiva) entre la variable dependiente y la variable "Grado de Desarrollo Comunal". Aquello quiere decir que existe evidencia estadística en estas cinco regiones para afirmar que uno de los factores que empíricamente determinan la asignación promedio del FNDR per cápita en las comunas de dichas regiones es su grado de desarrollo, siendo que en la medida de que una comuna es "más desarrollada" tiene un monto, en promedio, menor de asignación de FNDR per cápita.

En las regresiones con la variable "Log-Natural de la Inversión FNDR comunal per cápita" para cuatro regiones también se encontró evidencia que vincula la variable dependiente con la variable "Porcentaje del CORE elegido por una Provincia", sin embargo la dirección de dicha relación no es igual en todas las regresiones, lo que complejiza el análisis. Hay evidencia que en el caso de la Región de Antofagasta hay una relación positiva entre ambas variables, sin embargo en la Región de la Araucanía, la Región de Aysén y la Región de Magallanes, la relación entre ambas variables es negativa. Lo anterior implica que la hipótesis que relacionaba un mayor número de CORE elegido en una Provincia con la Inversión FNDR comunal per cápita, no solo no se acepta para estas tres regiones nombradas, sino que además se rechaza al encontrar evidencia estadística que avala justamente lo contrario. Sin embargo queda darle una explicación a dicha relación que contradice el resultado esperado de forma tan rotunda. Una explicación posible es que dentro del CORE de dichas regiones, ya sea de forma irreflexiva o formal a través de algún reglamento, todos o algunos de los consejeros apliquen algún criterio de compensación con las comunas que pertenecen a las provincias que eligen a menos consejeros, en base a algún criterio o factor que no haya sido incluido en este estudio como crecimiento económico, necesidades específicas de infraestructura o situaciones medioambientales. De cualquier modo este aspecto queda abierto para poder seguir explorando y comprendiendo las posibles causas de la relación en estas tres regiones.

Resulta particularmente llamativo el hecho de que las variables políticas, es decir la variable "Municipio con Apoyo del Gobierno" y particularmente la variable "Municipio con Apoyo CORE" no resultasen mostrar relaciones estadísticamente significativas para ninguna región bajo ningún formato de la variable dependiente, ni aún probando con distintos tramos de la serie de tiempo. Esto implica que, basados en los datos recolectados, no existe para ninguna región evidencia que corrobore la hipótesis de que la distribución comunal del FNDR, en términos brutos o per cápita, sea afectada por variables 
político partidarias.

En general, se puede concluir que en cada región se aplican criterios distintos, e incluso con distinto peso específico, para distribuir la asignación del FNDR. Aquella constatación empírica puede tener como explicaciones que las agendas sectoriales se impongan a la agenda regional, o que distintos gobiernos regionales tienen distintas capacidades para detectar las necesidades locales. De cualquier modo, un instrumento que pretende tener un rol de compensación territorial como el FNDR, para ser efectivo no solo debe cuidar los equilibrios interregionales, sino también intra-regionales o incluso inter-comunales, que como se ha constatado. Aquello sugiere la necesidad de reformular el FNDR, o de crear otro instrumento, que recoja esta dimensión, aumentando los grados de descentralización política y fiscal en las regiones y comunas.

Sin embargo quedan algunas aristas abiertas de análisis respecto al funcionamiento del FNDR como instrumento de inversión del Estado de carácter descentralizado. En primer lugar, el recurrir a fuentes de información respecto a pobreza, desigualdad, actividad económica, resultados electorales y desarrollo que puedan complementar y afinar los resultados aquí expuestos. En segundo lugar, el proceso político del Gobierno Regional, sigue siendo en buena parte opaco y su documentación a través de metodologías cualitativas tales como entrevistas o grupos focales permitiría cruzar la evidencia empírica generada con las motivaciones e intereses de los actores involucrados en el proceso y finalmente, analizar de forma empírica la relación entre Consejo Regional y otros organismos gubernamentales como las SEREMIS, la SUBDERE y el Ministerio de Desarrollo Social puede revelar disfunciones organizativas, sesgos metodológicos y asimetrías de poder que afecten negativamente el proceso de inversión del FNDR y del que el presente estudio sólo ha encontrado indicios.

\section{Referencias}

Acuña, E. (2009). Propuestas de Mejora al Diseño y Gestión del Fondo Nacional de Desarrollo Regional.

Aghón, G. (1993). Descentralización Fiscal: Marco Conceptual. CEPAL-GTZ, Santiago.

Aghón, G. (2001). Desarrollo Económico Local y Descentralización en América Latina: Análisis comparativo. CEPAL-GTZ, Santiago.

Barozet, E. (2006). Relecturas de la noción de clientelismo: una forma diversificada de intermediación política y social. Debate, (69):77-102.
Barozet, E. (2008). Populismo regional y Estado en Chile. EIAL, 19(2):45-60.

Blanco, I. y Gomá, R. (2003). Gobiernos locales y redes participativas: retos e innovaciones. Reforma y Democracia, (26).

Boiser, S. (1982). Política Económica, Organización Social y Desarrollo Regional. Cuadernos del ILPES, (26).

Boiser, S. (1992). El Conflicto Centro-Periferia en torno al Fondo Nacional de Desarrollo Regional. Serie Investigación, (02).

Boiser, S. (1997). El vuelo de una cometa. Una metáfora para una teoría del desarrollo territorial. Estudios Regionales, (47):41-79.

Boiser, S. (1999). El Desarrollo Territorial a Partir de la Construcción de Capital Sinergético. Una contribución al tema del capital intangible del desarrollo. En CEUR, editor, Instituciones y Actores del Desarrollo Territorial en el marco de la Globalización, pp. 273-297. Ediciones de la Universidad Bío-Bío, Santiago.

Cuervo, L. (2003). Evolución Reciente de las Disparidades Económicas Territoriales en América Latina: Estado del arte, recomendaciones de política y perspectivas de investigación. ILPES-CEPAL, Colección Gestión Pública NÂ41, Santiago.

Cuervo, L. (2006). Globalización y Territorio. ILPES-CEPAL, Serie Gestión Pública NÂำ, Santiago.

De la Maza, G. (2010). No Title.

Durnston, J. (2005). El Clientelismo Político en el Campo Chileno (Primera Parte): La democratización cuestionada. Revista Ciencias Sociales, II(1):1-30.

Elizalde, A. (2003). Planificación estratégica territorial y políticas públicas para el desarrollo local. ILPES-CEPAL, Serie Gestión Pública NÂ29, Santiago.

Finot, I. (2002). Descentralización y Participación en América Latina: Una mirada desde la economía. Revista de la CEPAL, (78):139-147.

Fresno, J. (2013). El Enfoque Territorial en los Futuros Fondos Estructurales. Journal Of Public Policies And Territories, (4):8-15.

Insunza, I. (1997). Descentralización de Recursos Hacia Los Gobiernos Regionales En Chile. CEPAL-GTZ, Serie Política Fiscal NÂ¹00, Santiago. 
Isunza, E. (2004). Interfaces Socio-estatales y Proyectos Políticos. La disputa entre rendición de cuentas y participación ciudadana desde la perspectiva de la igualdad compleja.

Letelier, L. (2012). Teoría y Práctica de la Descentralización Fiscal: El caso de la Ley de Rentas Municipales II. Ediciones UC, Santiago de Chile.

Miranda, L. (2008). La política clientelar de la política local. Tesis de magíster no publicada, Instituto de Iberoamérica, Universidad de Salamanca, Salamanca.

Monasterio, C. y Suárez, J. (1998). Manual de Hacienda Autonómica y Local. Ariel, Barcelona.

Organización para la Cooperación y el Desarrollo Económico (2009). Estudios Territoriales de la OCDE: Chile. OCDE-Ministerio del Interior, Santiago.

Palma, E. (2009). Los Desafíos de la Descentralización: La especificidad y las exigencias del caso chileno. En Pensando Chile desde la regiones. UFRO Ediciones, Temuco.

Prud'homme, R. (1995). The Dangers of Decentralization. Research Observer, (2):World Bank.

Schiling, M. (1999). Las Cuentas de la Descentralización. En Instituciones y Actores del Desarrollo
Territorial en el Marco de la globalización, pp. 43-67. Ediciones Universidad Bío-Bío, Santiago.

Schröter, B. (2010). Clientelismo Político Â¿Existe el fantasma y como se viste? Revista Mexicana de Sociología, I(72):141-175.

Silva, I. (2003). Disparidades, Competitividad Territorial y Desarrollo Local y Regional En América Latina. Technical report, Serie Gestión Pública $\mathrm{NA}^{\circ} 10$, Santiago.

Silva, I., Riffo, L., y González, S. (2012). La economía regional chilena en el período 1985-2009. ILPES-CEPAL, Serie Desarrollo Local NÂ10, Santiago de Chile.

Von Haldenwag, C. (1990). Hacia un Concepto Politológico de la Descentralización del Estado en América Latina. Revista FURE, XVI(50):61-77.

World Trade Organization, International Development Enterprises, y Japan External TradeOrganization (2011). Trade Patterns and global Value Chains in East Asia: From trade in good to trade in task. Secretearía de la WTO, Zurich.

Yin, R. K. (2003). Case Study Research: Design and Methods. Applied social research methods series, 5 . 